\title{
Review of: "Psychological distress, fear and coping among Malaysians during the COVID-19 pandemic"
}

Yong Zeng

Potential competing interests: The author(s) declared that no potential competing interests exist.

This study explored some of the risk and protective factors affecting the psychological pressure of the Malaysian public during the COVID-19 epidemic in the form of a questionnaire, and provided valuable suggestions for the Malaysian government in the future social recovery, so this is a meaningful investigation research.

There are a number of concerns with this study which include the following:

1.This study is to explore the general psychological problems of the Malaysian population. The sample size is still relatively small compared to the overall population and most of the subjects are concentrated in a certain area, so the representativeness of the sample is low.

2.The "study participants who drank alcohol in the last four weeks had lower levels of fear" described in the results part of this article conflicts with the explanation in the discussion part $\square$ those who drank alcohol in the past four weeks should not experience the same higher psychological distress with those who had higher levels of fear.

3.The overall writing logic also needs to be improved. There is a logical separation between the explanation of the discussion part and the results of the presentation, and there is a certain degree of confusion, so readers cannot be well convinced to agree with some of the author's views and conclusions. 